\title{
IS NONHELICAL HYDROMAGNETIC TURBULENCE PEAKED AT SMALL SCALES?
}

\author{
Nils Erland L. Haugen, ${ }^{1}$ Axel Brandenburg, ${ }^{2}$ and Wolfgang Dobler ${ }^{3}$ \\ Received 2003 June 25; accepted 2003 September 30; published 2003 October 24
}

\begin{abstract}
Nonhelical hydromagnetic turbulence without an imposed magnetic field is considered in the case where the magnetic Prandtl number is unity. The magnetic field is entirely due to dynamo action. The magnetic energy spectrum peaks at a wavenumber of about 5 times the minimum wavenumber in the domain, and not at the resistive scale, as has previously been argued. Throughout the inertial range, the spectral magnetic energy exceeds the kinetic energy by a factor of about 2.5 , and both spectra are approximately parallel. At first glance, the total energy spectrum seems to be close to $k^{-3 / 2}$, but there is a strong bottleneck effect and it is suggested that the asymptotic spectrum is $k^{-5 / 3}$. This is supported by the value of the second-order structure function exponent that is found to be $\zeta_{2}=0.70$, suggesting a $k^{-1.70}$ spectrum.
\end{abstract}

Subject headings: ISM: kinematics and dynamics — magnetic fields — MHD — turbulence

\section{INTRODUCTION}

It is generally accepted that in hydromagnetic turbulence the magnetic field tends to be more intermittent than the velocity field. This is evidenced by many numerical simulations where the magnetic field is dynamo-generated (Meneguzzi, Frisch, \& Pouquet 1981; Kida, Yanase, \& Mizushima 1991; Brandenburg et al. 1996; Kulsrud et al. 1997). Furthermore, linear theory predicts that the exponentially growing magnetic energy spectrum increases with wavenumber like $k^{+3 / 2}$ (Kazantsev 1968). Taken at face value, linear theory would suggest that the magnetic energy spectrum should be peaked at the "resistive" cutoff scale. In the case of the interstellar medium, the resistive cutoff scale would be tiny $\left(<10^{10} \mathrm{~cm}\right)$ in comparison with other relevant scales $\left(>10^{18} \mathrm{~cm}\right)$. There is, of course, no doubt that there are magnetic fluctuations in the interstellar medium at a scale below $10^{10} \mathrm{~cm}$, as evidenced by interstellar scintillation measurements (Goldreich \& Sridhar 1995), but it remains implausible that magnetic fields at such small scale contribute significantly to the energy budget.

The question of small-scale magnetic fields has worried theorists for the last decade. Kulsrud \& Anderson (1992) have shown that, independent of the possible presence of magnetic helicity, the kinematic magnetic energy spectrum follows the Kazantsev $k^{+3 / 2}$ law, and they speculate that this may suppress large-scale dynamo action (of $\alpha^{2}$ or $\alpha \Omega$ type; see also Vainshtein \& Cattaneo 1992). The case of helical dynamos is now reasonably well understood in the case of large magnetic Reynolds number. The saturation level of helical dynamos is not suppressed, but the saturation time is the resistive timescale (Brandenburg 2001). This is not the result of small-scale magnetic fields in general but due to the helical small-scale fields that are produced by the $\alpha$-effect as a by-product (Field \& Blackman 2002; Blackman \& Brandenburg 2002).

When the degree of kinetic helicity of the flow falls below a certain threshold, no large-scale dynamo action is possible and the magnetic energy spectrum is peaked at a scale much smaller than the forcing scale (Maron \& Blackman 2002). This may not be so much a concern for stellar dynamos where differential

\footnotetext{
${ }^{1}$ Department of Physics, Norwegian University of Science and Technology, Høyskoleringen 5, N-7034 Trondheim, Norway; nils.haugen@phys.ntnu.no.

${ }^{2}$ NORDITA, Blegdamsvej 17, DK-2100 Copenhagen Ø, Denmark; brandenb@nordita.dk.

${ }^{3}$ Kiepenheuer-Institut für Sonnenphysik, Schöneckstrasse 6, D-79104 Freiburg, Germany; dobler@kis.uni-freiburg.de.
}

rotation is important, but it could be a problem for dynamo action in the interstellar medium and in clusters of galaxies. Analytic approaches to the nonlinear saturation of nonhelical dynamos have corroborated the notion that the magnetic energy spectrum peaks at the resistive scale (Schekochihin et al. 2002a, 2002b). Numerical simulations have confirmed a peak well below the forcing scale (Cho \& Vishniac 2002a; Maron \& Cowley 2001), but a resolution of up to $256^{3}$ collocation points is still insufficient to establish whether the location of the peak is different from the resistive scale.

So far there has been no evidence for $k^{-5 / 3}$ inertial range scaling of the magnetic energy as in the case of an imposed field (Goldreich \& Sridhar 1995; Cho \& Vishniac 2000b; Maron \& Goldreich 2001) or as in decaying hydromagnetic turbulence (Biskamp \& Müller 2000). A difficulty in establishing power-law scaling is the lack of a sufficiently long inertial range. In some cases, the use of hyperresistivity (where the $\nabla^{2}$ diffusion operator is replaced, for example, by $-\nabla^{4}$, in order to shorten the diffusive subrange) has led to the concern that it may cause an artificially enhanced "bottleneck effect" with a shallower $k^{-1}$ spectrum just before the dissipative subrange (Biskamp \& Müller 2000). Yet another problem is the possibility of a physical bottleneck effect (Falkovich 1994) that will be more extreme in three-dimensional spectra than in the one-dimensional spectra accessible from turbulence experiments (Biskamp \& Müller 2000; Dobler et al. 2003).

In this Letter, we use simulations at resolutions of up to $1024^{3}$ meshpoints to study the form of the magnetic and kinetic energy spectra in the inertial range in the case where the turbulence is driven at large scales and the magnetic field is selfgenerated. No hyperviscosity or hyperresistivity is used.

\section{EQUATIONS}

Here we consider subsonic turbulence in an isothermal electrically conducting gas with constant sound speed $c_{s}$ in a periodic box of size $2 \pi \times 2 \pi \times 2 \pi$. The governing equations are

$$
\frac{D \boldsymbol{u}}{D t}=-c_{s}^{2} \boldsymbol{\nabla} \ln \rho+\frac{\boldsymbol{J} \times \boldsymbol{B}}{\rho}+\boldsymbol{F}_{\mathrm{visc}}+\boldsymbol{f}
$$

where $D / D t=\partial / \partial t+\boldsymbol{u} \cdot \boldsymbol{\nabla}$ is the advective derivative, $\boldsymbol{J}=$ 


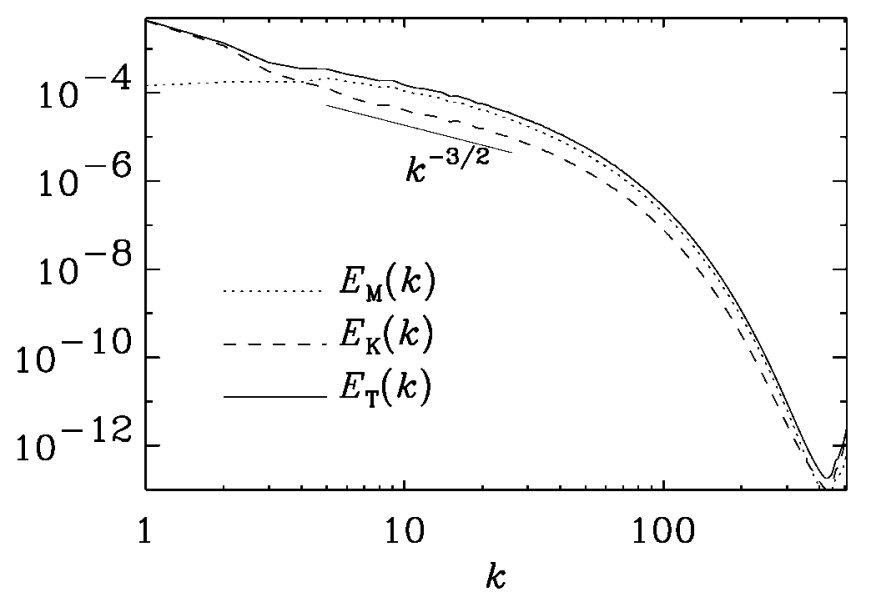

FIG. 1.-Magnetic, kinetic, and total energy spectra; $1024^{3}$ meshpoints. The Reynolds number is $u_{\mathrm{rms}} / \nu k_{f} \approx 960$.

$\boldsymbol{\nabla} \times \boldsymbol{B} / \mu_{0}$ is the current density, $\boldsymbol{B}$ is the magnetic field, $\mu_{0}$ is the vacuum permeability, and

$$
\boldsymbol{F}_{\mathrm{visc}}=\nu\left(\nabla^{2} \boldsymbol{u}+\frac{1}{3} \boldsymbol{\nabla} \boldsymbol{\nabla} \cdot \boldsymbol{u}+2 \mathcal{S} \cdot \boldsymbol{\nabla} \ln \rho\right)
$$

is the viscous force, where $\nu=$ const is the kinematic viscosity, $\mathcal{S}_{i j}=\frac{1}{2}\left(u_{i, j}+u_{j, i}\right)-\frac{1}{3} \delta_{i j} u_{k, k}$ is the traceless rate of strain tensor, and $\boldsymbol{f}$ is a random forcing function (see below). The continuity equation is written in terms of logarithmic density,

$$
\frac{D \ln \rho}{D t}=-\boldsymbol{\nabla} \cdot \boldsymbol{u}
$$

and the induction equation is solved in terms of the magnetic vector potential $\boldsymbol{A}$, where $\boldsymbol{B}=\boldsymbol{\nabla} \times \boldsymbol{A}$,

$$
\frac{\partial \boldsymbol{A}}{\partial t}=\boldsymbol{u} \times \boldsymbol{B}+\eta \nabla^{2} \boldsymbol{A}
$$

and $\eta=$ const is the magnetic diffusivity (or resistivity); we choose $\eta=\nu$-i.e., our magnetic Prandtl number is unity. We adopt a forcing function $\boldsymbol{f}$ of the form

$$
\boldsymbol{f}(\boldsymbol{x}, t)=\operatorname{Re}\left\{N f_{\boldsymbol{k}(t)} \exp [i \boldsymbol{k}(t) \cdot \boldsymbol{x}+i \phi(t)]\right\},
$$

where $\boldsymbol{x}=(x, y, z)$ is the position vector and $-\pi \leq \phi(t)<\pi$ is a ( $\delta$-correlated) random phase. The normalization factor is $N=f_{0} c_{s}\left(k c_{s} / \delta t\right)^{1 / 2}$, with $f_{0}$ a nondimensional forcing amplitude, $k=|\boldsymbol{k}|$, and $\delta t$ the length of the time step; we chose $f_{0}=0.02$ so that the maximum Mach number stays below about 0.5 (the rms Mach number is close to 0.12 in all runs. The vector $f_{k}$ describes nonhelical transversal waves with $\left|\boldsymbol{f}_{\boldsymbol{k}}\right|^{2}=1$ and $\boldsymbol{f}_{\boldsymbol{k}}=(\boldsymbol{k} \times \boldsymbol{e}) /\left[\boldsymbol{k}^{2}-(\boldsymbol{k} \cdot \boldsymbol{e})^{2}\right]^{1 / 2}$, where $\boldsymbol{e}$ is an arbitrary unit vector. At each time step, we select randomly one of 20 possible wavevectors in the range $1 \leq|\boldsymbol{k}|<2$ around the forcing wavenumber, $k_{f}=1.5$.

The equations are solved using the same method as in Brandenburg (2001), but here we employ a new cache and memory

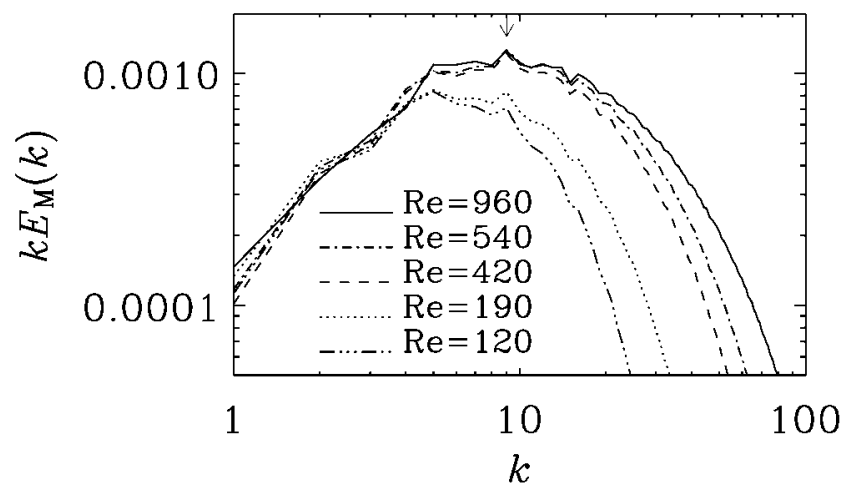

FIG. 2.-Spectral magnetic energy per logarithmic wavenumber interval for runs with different Reynolds numbers. The arrow corresponds to the peak $(k=9)$ of the four largest runs.

efficient $\operatorname{code}^{4}$ using Message Passing Interface library calls for communication between processors.

\section{RESULTS}

In Figure 1, we plot magnetic, kinetic, and total energy spectra, $E_{M}(k), E_{K}(k)$, and $E_{T}=E_{M}+E_{K}$, respectively, for our largest resolution run with $1024^{3}$ meshpoints. ${ }^{5}$ The magnetic energy displays a nearly flat spectrum in the range $1 \leq k \leq 5$, peaks at $k \approx 5$, and begins to show an inertial range in $8 \leq k \leq 25$, followed by a dissipative subrange over one decade. In the inertial range, $E_{M}(k) / E_{K}(k)$ is about 2.5. A plot of magnetic energy per logarithmic wavenumber interval, $k E_{M}(k)$, shows that most of the magnetic energy comes from a band of wavenumbers around $k=9$-independent of the Reynolds number once the latter is above $\sim 400$ (see Fig. 2).

The energy ratio in the inertial range is similar to the ratio of the dissipation rates of magnetic and kinetic energies, $\epsilon_{M} / \epsilon_{K}$, which is about 2.3; see Figure 3, which shows that magnetic and kinetic energy dissipation rates approach $70 \%$ and $30 \%$, respectively, of the total dissipation rate, $\epsilon_{T}=\epsilon_{M}+\epsilon_{K}$. The convergence of relative dissipation rates is compatible with Kolmogorov's concept of a constant, scale-independent energy flux across the spectrum, which seems to apply also separately for velocity and magnetic fields. This picture would be difficult to reconcile if the magnetic energy were to always peak at the resistive scale. We emphasize that the inertial range is not representative of the total energy, which, in turn, is dominated by small wavenumbers. The ratio of total magnetic to kinetic energies is only 0.4 and seems again to be asymptotically independent of Reynolds number (Fig. 3).

By comparing runs at different resolution, one can clearly see that in the range $3 \leq k \leq 20$ the total energy spectrum is shallower than $k^{-5 / 3}$ (Fig. 4). This could perhaps be due to the bottleneck effect that is known to exist also in wind tunnel turbulence, where it has been described by a weak $k^{-1}$ contribution (She \& Jackson 1993). There are several reasons why such a bottleneck effect might occur. First, recent studies by Dobler et al. (2003) have shown that the bottleneck effect is much stronger in shell-integrated three-dimensional spectra

\footnotetext{
${ }^{4}$ We use the Pencil Code, which is a cache efficient grid-based high-order code (sixth order in space and third order in time) for solving the compressible MHD equations; http://www.nordita.dk/data/brandenb/pencil-code.

${ }^{5}$ These spectra are, as usual, integrated over shells in $k$-space and normalized such that $\int E_{K} d k=\frac{1}{2}\left\langle\boldsymbol{u}^{2}\right\rangle$ and $\int E_{M} d k=\frac{1}{2}\left\langle\boldsymbol{B}^{2}\right\rangle / \mu_{0}$.
} 

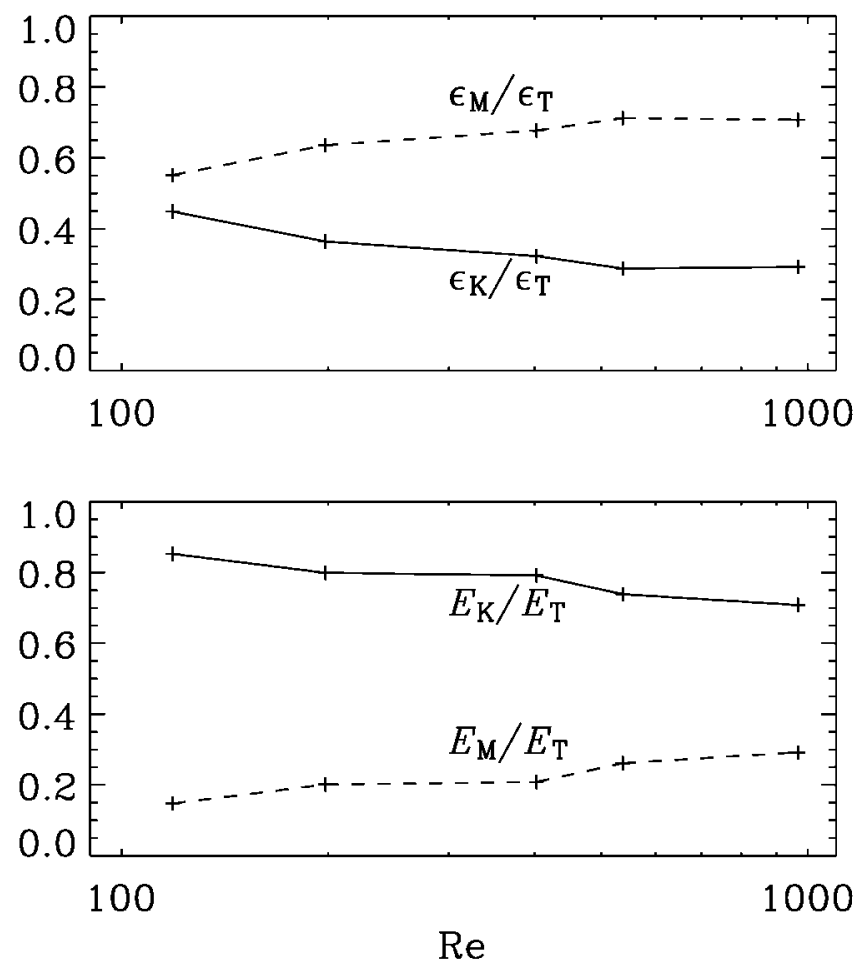

FIG. 3.-Approximate convergence of relative magnetic and kinetic energy dissipation rates (upper panel) and the relative energies (lower panel) as a function of Reynolds number.

compared to just longitudinal or transversal one-dimensional spectra available in wind tunnel turbulence. Second, the bottleneck effect may simply be stronger for hydromagnetic turbulence due to the dynamo effect that is expected to produce magnetic energy preferentially at $k \approx k_{f} R_{c}^{1 / 2} \approx 5 \ldots 10$, where $R_{c} \approx 30$ is the critical magnetic Reynolds number for dynamo action (Subramanian 1999). Third, numerical effects such as hyperdiffusion or other effects causing departures from the physical $\nabla^{2}$ diffusion operator could cause an artificial bottleneck effect (Biskamp \& Müller 2000; Biskamp, Schwarz, \& Celani 1998), which may also explain the extended $k^{-1}$ range in compressible nonmagnetic simulations using the piecewise parabolic method (Porter, Woodward, \& Pouquet 1998).

We believe that numerical effects do not cause such artifacts in our simulations, because we use the physical $\nabla^{2}$ diffusion operator and our discretization is accurate to sixth order in space and third order in time. We have compared two runs with identical values of $\nu$ and $\eta$, one with $256^{3}$ meshpoints and the other one with $512^{3}$, and found the same spectra (Haugen, Brandenburg, \& Dobler 2003). We have also compared with runs using double precision and found the spectra to be the same. The runs with up to $512^{3}$ meshpoints have run for up to 80 turnover times, $\left(u_{\mathrm{rms}} k_{f}\right)^{-1}$, which should be long enough to eliminate transients. The run with $1024^{3}$ meshpoints has only run for five turnover times, but the results are otherwise in qualitative agreement with those of the $512^{3}$ run.

By comparing with nonmagnetic runs at the same resolution $\left(1024^{3}\right.$ meshpoints), we have found that in hydromagnetic turbulence the bottleneck effect is about equally strong. This leaves us with the possibility that the bottleneck effect is real, both for hydrodynamic and for hydromagnetic turbulence, but that it is simply more pronounced in fully three-dimensional

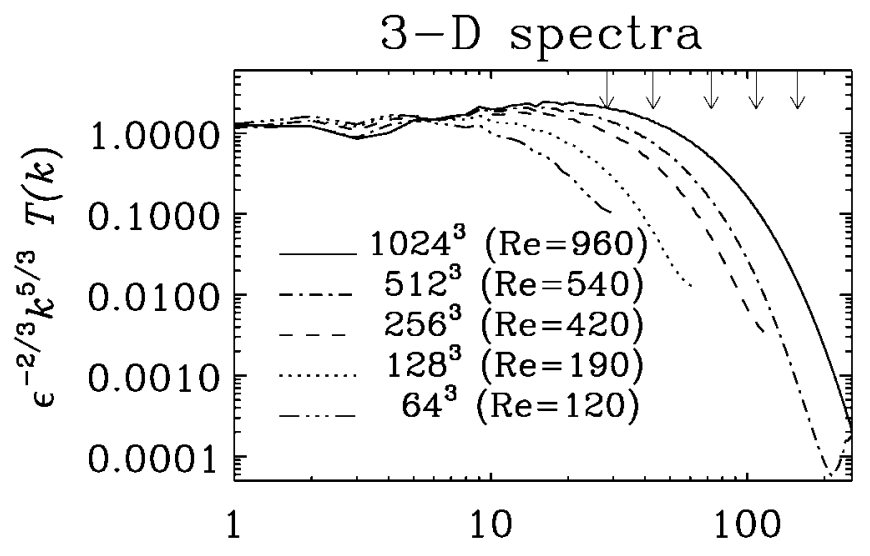

FIG. 4.-Total energy spectra compensated by $\epsilon_{T}^{-2 / 3} k^{5 / 3}$, where $\epsilon_{T}$ is the rate of total energy dissipation. Spectra for different resolution are compared. The dissipation cutoff wavenumber, $k_{d}=\left(\epsilon_{T} / \nu^{3}\right)^{1 / 4}$, is indicated by short arrows at the top of the plot. The Reynolds number, $\operatorname{Re}=u_{\mathrm{rms}} / \nu k_{f}$, is given in the legend.

spectra. It is therefore still possible that at larger Reynolds numbers the true inertial range spectrum will have a $k^{-5 / 3}$ behavior both for kinetic and magnetic energies (Goldreich \& Sridhar 1995).

Alternatively, a $k^{-3 / 2}$ spectrum might be readily explicable in terms of the Iroshnikov-Kraichnan phenomenology (Iroshnikov 1963; Kraichnan 1965). It does of course ignore local anisotropy, but more importantly, it predicts that the fourth-order structure function, $S_{4}(l)$, scales linearly in the inertial range, $\zeta_{4} \equiv$ $d \ln S_{4} / d \ln l=1$ (see Biskamp 1993). To assess this possibility, we calculate the double-logarithmic derivative, $\zeta_{p}=$ $d \ln S_{p} / d \ln l$, of the unsigned structure function, $S_{p}=\left\langle\left|z^{ \pm}\right|^{p}\right\rangle$, where $z^{ \pm}=\boldsymbol{u} \pm \boldsymbol{B} / \sqrt{\mu_{0} \rho}$ are the Elsasser variables. The scalings of $z^{+}$and $z^{-}$turn out to be similar, so we take the average value.

Figure 5 shows that our data are inconsistent with IroshnikovKraichnan scaling $\left(\zeta_{4} \approx 1.3\right.$, rather than 1$)$. Instead, our data are consistent with $\zeta_{3}=1$ in the inertial range (Fig. 6). Note that linear inertial range scaling of $S_{3}(l)$, i.e., $\zeta_{3}=1$, is an exact result for hydrodynamic turbulence (Frisch 1995). Using the extended self-similarity hypothesis (Benzi 1993), we plot $S_{p}(l)$ against $S_{3}(l)$ to obtain a more accurate determination of $\zeta_{p}$ for

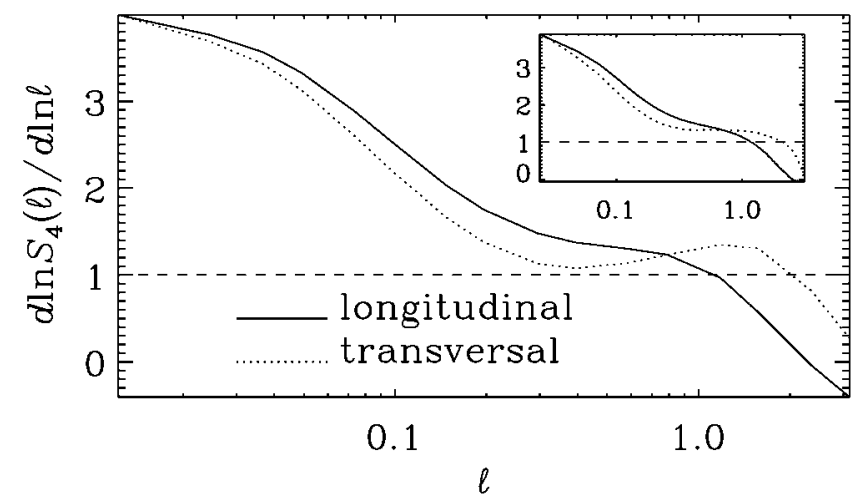

FIG. 5.-Fourth-order structure function for runs with $512^{3}$ meshpoints. Clearly, $S_{4}(l)$ is not compatible with linear scaling. The inset gives the result for $256^{3}$ meshpoints. The scaling for transversal structure functions (dotted lines) tends to be better than for the longitudinal ones (solid lines). The statistics for the $256^{3}$ runs is somewhat better than for the shorter $512^{3}$ runs. 


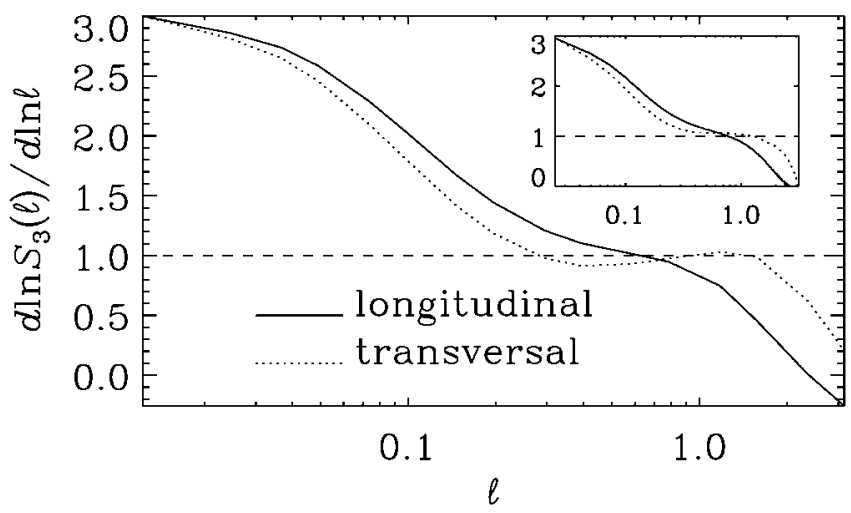

FIG. 6.-Same as Fig. 5, but for $S_{3}(l)$. Note that $S_{3}(l)$ is compatible with linear scaling, i.e., $\zeta_{3}=1$ in the inertial range.

$p \neq 3$. Our results are compatible with She \& Leveque (1994) and hence also with earlier forced simulations with an external field (Cho, Lazarian, \& Vishniac 2002a). In particular, $\zeta_{2}+1$ is the negative slope of the total energy spectrum: we find $\zeta_{2}=$ 0.70 , which is again in agreement with Kolmogorov scaling. This agrees also with simulations of decaying helical turbulence (Biskamp \& Müller 2000), except that there the higher moments have been found to be smaller.

\section{CONCLUSIONS}

For nonhelically forced hydromagnetic turbulence, a resolution of $1024^{3}$ is necessary in order to begin to establish inertial range scaling. Nevertheless, the energy spectra show what we interpret as a strong bottleneck effect. This effect is particularly strong in the shell-integrated spectra. Throughout the inertial range, however, the magnetic energy exceeds the kinetic energy by a factor of about 2.5. Both kinetic and magnetic energies are dominated by the spectral values at the beginning of the inertial range and independent of magnetic resistivity. The values $\zeta_{2}=0.70$ and $\zeta_{3}=1.0$ strongly favor an asymptotic $k^{-5 / 3}$ spectrum.

Our results do not support recent claims that the magnetic energy spectrum peaks at the resistive scale (Maron \& Cowley 2001; Maron \& Blackman 2002; Schekochihin et al. 2002a, 2002 b). However, closer inspection of Figure 3 of Maron \& Cowley (2001) or Figure 1 of Maron \& Blackman (2002) reveals that also in their cases the magnetic energy peaks at about 5 , in agreement with our results. This is comparable to the value $k_{f} R_{c}^{1 / 2} \approx 8$ expected based on a nonlinear closure model (Subramanian 1999). Here $R_{c} \approx 30$ is the critical magnetic Reynolds number for dynamo action (Haugen et al. 2003), and $k_{f} \approx 1.5$. For $k \geq 8$, we find the spectrum to be Kolmogorov-like.

In the interstellar medium, the magnetic Prandtl number is very large (Kulsrud \& Anderson 1992). This is also the regime in which the claims regarding the peak at the resistive scale are thought to apply best. On the other hand, preliminary results suggest that even for a magnetic Prandtl number between 5 and 30 the magnetic energy spectrum still peaks at $k \approx 5$ (Haugen et al. 2003). However, the spectrum shows now a possible $k^{-1}$ tail near the viscous dissipation range where ohmic dissipation is still weak (Cho, Lazarian, \& Vishniac 2002b; Haugen et al. 2003). In the interstellar medium, even though the magnetic Prandtl number is very large, the Reynolds number is also very large, so the viscous cutoff scale is still small $\left(\sim 10^{16} \mathrm{~cm}=\right.$ $0.003 \mathrm{pc})$. We expect that in the range $0.01 \ldots 10 \mathrm{pc}$ kinetic and magnetic energy spectra are parallel and show $k^{-5 / 3}$ scaling, but currently the numerical resolution is still insufficient to demonstrate this.

We thank Jason Maron, Åke Nordlund, and Kandaswamy Subramanian for their comments on this Letter. Use of the parallel computers in Trondheim (Gridur), Odense (Horseshoe), and Leicester (Ukaff) is acknowledged.

\section{REFERENCES}

Benzi, R., Ciliberto, S., Tripiccione, R., Baudet, C., Massaioli, F., \& Succi, S. 1993, Phys. Rev. E, 48, 29

Biskamp, D. 1993, Nonlinear Magnetohydrodynamics (Cambridge: Cambridge Univ. Press)

Biskamp, D., \& Müller, W.-C. 2000, Phys. Plasmas, 7, 4889

Biskamp, D., Schwarz, E., \& Celani, A. 1998, Phys. Rev. Lett., 81, 4855

Blackman, E. G., \& Brandenburg, A. 2002, ApJ, 579, 359

Brandenburg, A. 2001, ApJ, 550, 824

Brandenburg, A., Jennings, R. L., Nordlund, Å., Rieutord, M., Stein, R. F., \& Tuominen, I. 1996, J. Fluid Mech., 306, 325

Cho, J., Lazarian, A., \& Vishniac, E. T. 2002a, ApJ, 564, 291 2002b, ApJ, 566, L49

Cho, J., \& Vishniac, E. T. 2000a, ApJ, 538, 217 2000b, ApJ, 539, 273

Dobler, W., Haugen, N. E. L., Yousef, T. A., \& Brandenburg, A. 2003, Phys. Rev. E, 68, 026304

Falkovich, G. 1994, Phys. Fluids, 6, 1411

Field, G. B., \& Blackman, E. G. 2002, ApJ, 572, 685

Frisch, U. 1995, Turbulence: The Legacy of A. N. Kolmogorov (Cambridge: Cambridge Univ. Press)

Goldreich, P., \& Sridhar, S. 1995, ApJ, 438, 763
Haugen, N. E. L., Brandenburg, A., \& Dobler, W. 2003, preprint (astro-ph/ 0307059)

Iroshnikov, R. S. 1963, Soviet Astron., 7, 566

Kazantsev, A. P. 1968, Soviet Phys.-JETP, 26, 1031

Kida, S., Yanase, S., \& Mizushima, J. 1991, Phys. Fluids A, 3, 457

Kraichnan, R. H. 1965, Phys. Fluids, 8, 1385

Kulsrud, R. M., \& Anderson, S. W. 1992, ApJ, 396, 606

Kulsrud, R. M., Cen, R., Ostriker, J. P., \& Ryu, D. 1997, ApJ, 480, 481

Maron, J., \& Blackman, E. G. 2002, ApJ, 566, L41

Maron, J., \& Cowley, S. C. 2001, preprint (astro-ph/0111008)

Maron, J., \& Goldreich, P. 2001, ApJ, 554, 1175

Meneguzzi, M., Frisch, U., \& Pouquet, A. 1981, Phys. Rev. Lett., 47, 1060

Porter, D. H., Woodward, P. R., \& Pouquet, A. 1998, Phys. Fluids, 10, 237

Schekochihin, A. A., Cowley, S. C., Hammett, G. W., Maron, J. L., \& McWilliams, J. C. 2002a, New J. Phys., 4, 84

Schekochihin, A. A., Maron, J. L., Cowley, S. C., \& McWilliams, J. C. 2002b, ApJ, 576, 806

She, Z.-S., \& Jackson, E. 1993, Phys. Fluids, A5, 1526

She, Z.-S., \& Leveque, E. 1994, Phys. Rev. Lett., 72, 336

Subramanian, K. 1999, Phys. Rev. Lett., 83, 2957

Vainshtein, S. I., \& Cattaneo, F. 1992, ApJ, 393, 165 